CLINICAL STUDY

\title{
Plasma omentin-1 levels are reduced in non-obese women with normal glucose tolerance and polycystic ovary syndrome
}

\author{
Ji-Hun Choi ${ }^{1}$, Eun-Jung Rhee ${ }^{1, *}$, Kye-Hyun Kim ${ }^{2, *}$, Hee-Yeon Woo ${ }^{3}$, Won-Young Lee ${ }^{1}$ and Ki-Chul Sung ${ }^{4}$ \\ ${ }^{1}$ Division of Endocrinology and Metabolism, ${ }^{2}$ Department of Obstetrics and Gynecology, ${ }^{3}$ Department of Laboratory Medicine and ${ }^{4}$ Division of Cardiology, \\ Department of Internal Medicine, Kangbuk Samsung Hospital, Sungkyunkwan University School of Medicine, Seoul 110-746, Korea \\ (Correspondence should be addressed to E-J Rhee; Email: hongsiri@hanmail.net, K-H Kim; Email: khmd.kim@samsung.com)
}

*(E-J Rhee and K-H Kim contributed equally to this work)

\begin{abstract}
Objective: Omentin-1 is a novel adipokine that increases insulin sensitivity and is expressed in visceral adipose tissue. The aim of this study was to determine the metabolic parameters that influence plasma omentin-1 levels in women with polycystic ovary syndrome (PCOS).

Design and methods: A cross-sectional study was performed in 87 women with PCOS and 53 body mass index (BMI)-matched healthy controls including 39 non-obese, normal-weight (NW) PCOS women with normal glucose tolerance (NGT) and 44 BMI- and homeostasis model assessment (HOMA)matched controls. Indices of insulin sensitivity, metabolic variables, circulating androgen levels, serum adiponectin, and omentin-1 levels were measured. A $75 \mathrm{~g}$ oral glucose tolerance test was performed in all participants.

Results: Plasma omentin-1 levels were significantly lower in women with PCOS compared with those in BMI-matched controls $(P<0.001)$. A significantly lower level of plasma omentin-1 was observed in non-obese women with PCOS and NGT compared with that in BMI- and HOMA-matched controls $(P<0.001)$. Omentin-1 level was negatively correlated with BMI, indices of insulin sensitivity, and circulating androgens and was associated with greater $2 \mathrm{~h}$ postprandial glucose, C-peptide, and insulin levels compared with fasting values. Within the NW and NGT groups, omentin-1 levels remained negatively correlated with BMI, $2 \mathrm{~h}$ postprandial C-peptide, and circulating androgens and demonstrated a negative linear trend according to quartile of free testosterone $(P=0.028)$.

Conclusions: Plasma levels of omentin-1 were reduced in non-obese women with PCOS and NGT. Postprandial hyperinsulinemia and hyperglycemia contributed more to lower omentin-1 levels than did fasting values in the setting of PCOS. Increased androgen levels contributed to decreased omentin-1 levels in women with PCOS.
\end{abstract}

European Journal of Endocrinology 165 789-796

\section{Introduction}

Polycystic ovary syndrome (PCOS), a common endocrine-metabolic reproductive disorder affecting $\sim 6-10 \%$ of reproductive aged women, is characterized by irregular menses (IM) and hyperandrogenism (HA) $(1,2)$. It is frequently associated with multiple risk factors for cardiovascular diseases (CVDs), such as insulin resistance (IR), the development of compensatory hyperinsulinemia, impaired glucose regulation (IGR), dyslipidemia, and visceral obesity (3-8). IR in PCOS is due in part to the high prevalence of obesity in those with the disorder. Nonetheless, $\sim 40 \%$ of women with PCOS, even in the United States, are not obese (1). Though controversy still exists regarding whether IR results from PCOS or from the concurrent obesity (9), it is possible that adipose tissue dysfunction may play a role in the observed IR and, consequently, to the metabolic and cardiovascular consequences of the disorder. Evidence exists for altered adipocyte function in PCOS, with studies reporting altered secretion of adipokines, such as adiponectin (10).

Adipose tissue is an endocrine organ that has widespread effects on carbohydrate and lipid metabolism. Furthermore, it is apparent that accumulation of visceral adipose tissue poses a greater cardiometabolic risk than does subcutaneous adipose tissue (11), as removal of visceral but not subcutaneous adipose tissue has been demonstrated to improve insulin sensitivity (12). Omentin-1 is a novel fat depot-specific adipokine that was identified from a cDNA library of visceral omental adipose tissue by Yang et al. (13) and is preferentially produced by visceral adipose tissue compared with subcutaneous adipose tissue. In vitro experiments have revealed that treatment with recombinant omentin-1 enhanced insulin-stimulated glucose uptake in human 
subcutaneous and omental adipocytes, triggering Akt signaling in both the absence and the presence of insulin (13). Furthermore, plasma omentin-1 level was inversely correlated with obesity and IR (14).

PCOS is known as a pre-diabetic state and is associated with a higher prevalence of obesity. However, Asian women with PCOS are less likely to have metabolic dysfunction, IR, and obesity compared with Caucasian women with PCOS (15-17). There is little data on omentin levels within the context of PCOS (18, 19). To our knowledge, no prior studies have addressed omentin levels in non-obese women with PCOS and normal glucose tolerance (NGT).

In this study, we measured plasma omentin-1 levels in Korean women with PCOS and compared them with those of body mass index (BMI)-matched controls to evaluate their relationship, as well as their correlations with BMI, IR, and androgen secretion. This study also investigated contributing factors, other than BMI and IR, to the regulation of plasma omentin-1 in women with PCOS. To explore potential contributing factors, plasma omentin-1 levels in a second cohort of nonobese women with PCOS and NGT were compared with those of a control group matched for BMI, IR stratification according to BMI, glucose metabolism status, and free testosterone.

\section{Subjects and methods}

\section{Study population}

In total 140 premenopausal Korean women with $(n=87)$ and without $(n=53)$ PCOS were recruited from the Endocrinology and Gynecology Clinics in the Kangbuk Samsung Hospital, Seoul, Korea. Control subjects were healthy volunteers recruited through advertisements within the hospital. PCOS was defined as the presence of at least two of the following three symptoms, after exclusion of other etiologies, such as, congenital adrenal hyperplasias, androgen-secreting tumors, Cushing's syndrome, according to the Rotterdam criteria (2): i) oligo- and/or anovulation, ii) clinical and/or biochemical signs of HA, and iii) polycystic ovaries present during transvaginal ultrasound examination. Clinical HA was defined by a modified Ferriman and Gallwey score of more than eight (20), and biochemical HA was defined as an elevation of serum testosterone levels above 97.5 percentile limits, which is $>0.6 \mathrm{ng} / \mathrm{ml}$ for total testosterone level and $2.07 \mathrm{ng} / \mathrm{ml}$ for free testosterone level. When the subjects with PCOS were divided into four subgroups, the proportions in the individual groups are as follows (15): i) the IM/HA/PCO group $(n=43,491 \%)$, ii) the IM/PCO group $(n=34$, $39.6 \%)$, iii) the IM/HA group ( $n=9,9.8 \%)$, and iv) the HA/PCS group $n=1,1.5 \%$ ). Also, the presence of other endocrinopathies that could have potentially caused these phenotypes was ruled out. Control subjects were women with regular menstrual cycles that had no evidence of hirsutism, acne, alopecia, or endocrine dysfunction. Exclusion criteria for the study included age $>40$ years, known CVD, thyroid disease, neoplasms, current tobacco use, hypertension (blood pressure (BP) of $140 / 90 \mathrm{mmHg}$ or greater), and renal impairment (serum creatinine $>120 \mu \mathrm{mol} / \mathrm{l}$ ). No study subjects had taken any medications for at least 6 months prior to the study, including oral contraceptives, glucocorticoids, ovulation induction agents, anti-obesity drugs, estrogenic, anti-androgenic, or antihypertensive medications. The exclusion of other disorders with similar clinical symptoms was done by performing appropriate tests. The same exclusion criteria applied to both cases and controls. The study protocol was approved by the Ethics Committee of Kangbuk Samsung Hospital, Sungkyunkwan University. All participants provided written informed consent for participation.

To determine the influence of PCOS on omentin-1 level at different stages of obesity, we subdivided both PCOS and controls into three groups according to the International Obesity Task Force (IOTF) and BMI thresholds for obesity in adult Asians as proposed by the WHO (21). Women with PCOS were subdivided into a lean group, BMI $<18.5 \mathrm{~kg} / \mathrm{m}^{2}(n=17)$, a normal weight group (NW), BMI $>18.5$ and $<23 \mathrm{~kg} / \mathrm{m}^{2}(n=50)$, and an overweight and obese group, BMI $\geq 23 \mathrm{~kg} / \mathrm{m}^{2}(n=20)$. The control group contained eight women with BMI $<18.5 \mathrm{~kg} / \mathrm{m}^{2}$, 41 women with a BMI between 18.5 and $23 \mathrm{~kg} / \mathrm{m}^{2}$, and four women with BMI $\geq 23 \mathrm{~kg} / \mathrm{m}^{2}$. In addition, subjects in both the PCOS and the control groups were also stratified into two subgroups according to glucose metabolism status as assessed by a $75 \mathrm{~g}$ oral glucose tolerance test (OGTT) (22). In the PCOS group, 44 subjects had normal glucose regulation (NGR) and 43 subjects had IGR, including impaired fasting glucose (IFG), impaired glucose tolerance (IGT), and newly diagnosed, and as yet untreated type 2 diabetes mellitus. In the control group, 47 subjects had NGR and six subjects had IGR, including IFG and IGT. A second nested case-control cohort of nonobese (BMI $<23 \mathrm{~kg} / \mathrm{m}^{2}$ ) women with NGT was formed among the original study population, in which a subset of 83 subjects was matched in a case-control manner for BMI, homeostasis model assessment-IR (HOMA-IR) index, composed of 39 PCOS and 44 control subjects.

\section{Clinical and biochemical measurements}

Clinical variables such as weight, height, and BP were assessed in all subjects using standard protocols during outpatient visit to the hospital. The BMI was calculated as weight divided by the square of height $\left(\mathrm{kg} / \mathrm{m}^{2}\right)$. Subjects presenting to the Gynecology Clinics following an 8-12 h overnight fast underwent fasting baseline laboratory tests and a $75 \mathrm{~g}$ OGTT. Plasma glucose, C-peptide, and insulin levels were measured following fasting and $2 \mathrm{~h}$ after oral glucose load. Fasting blood samples were obtained for analysis of plasma hormones, 
fatty acids, and adipokines. The hexokinase method was used to measure glucose levels, and an enzymatic colorimetric test was used to measure the total cholesterol, triglyceride (TG), high-density lipoprotein cholesterol (HDL-cholesterol), and low-density lipoprotein cholesterol (LDL-cholesterol) levels. Insulin was measured using an IRMA (Biosource, Nivelles, Belgium). C-peptide was measured using a RIA method with a commercial kit (BioSource, Europe S.A.). The HOMA-IR was derived from calculations according to the following formula: fasting insulin $(\mathrm{mU} / \mathrm{ml}) \times$ fasting glucose $(\mathrm{mmol} / \mathrm{l}) / 22.5$ (23). High sensitivity C-reactive protein levels were measured using immunonephelometry (Dade Behering Co., Marburg, Germany).

\section{Measurement of plasma hormones and adipokines}

Women with PCOS and control subjects were evaluated for serum total testosterone, free testosterone, 17-OH-progesterone, DHEA-sulphate, and sex hormone-binding globulin (SHBG) using RIA methods (Siemens, Los Angeles, CA, USA). The free androgen index (FAI) was calculated as total testosterone/SHBG $\times 100$. The plasma levels of omentin-1 (BioVendor, Candler, NC, USA) and adiponectin (R\&D Systems, Minneapolis, MN, USA) were measured using commercially available ELISA according to the manufacturer's protocols. In all hormonal assays, intra- and inter-assay coefficients of variation (CV) for all analytes were $<10 \%$.

\section{Statistical analyses}

Data were analyzed using SPSS Software (Version 17, SPSS, Inc., Chicago, IL, USA). All data are presented as mean \pm s.D., mean (S.E.M.), or median with interquartile range. The distribution of continuous variables was evaluated with log transformations as required. Differences between two groups were assessed using Student's $t$-test or the Mann-Whitney $U$ test. Data involving more than two groups were assessed using one-way ANOVA with the Tukey's post hoc analysis. Omentin-1 levels were compared using analysis of covariance (ANCOVA) to correct for age, BMI, and HOMA-IR. Spearman's rank correlation test was used for calculation of associations between variables. Logistic regression analysis was performed to determine the best model to predict development of PCOS. A $P$ value $<0.05$ was considered statistically significant.

\section{Results}

\section{Women with PCOS had reduced omentin-1 levels}

Table 1 includes the anthropometric, biochemical, and hormonal data for the women with PCOS and the normal controls. There were no significant differences in BMI between subjects with PCOS and controls $(P=0.124)$, despite the control group being slightly older than the PCOS group. Women with PCOS had higher glucose, C-peptide, HOMA-IR, TG, HDL-cholesterol, and insulin levels (in both the fasting state and $2 \mathrm{~h}$ following an OGTT) compared with those of the BMImatched control group $(P<0.05)$. Although fasting insulin levels tended to be higher in women with PCOS than they were in the controls, this difference was not significant $(P=0.057)$.

Women with PCOS had higher testosterone and FAI levels with lower SHBG levels compared with those of the controls $(P<0.001)$. ELISA analysis of plasma omentin-1 levels revealed that women with PCOS had significantly lower omentin-1 levels compared with those of BMI-matched control subjects $(P<0.001$; Table 1, Fig. 1A). Although adiponectin levels tended to be lower in women with PCOS than they were in the controls, these differences were not significant (Table 1).

As part of the study design, the subjects in the second cohort were non-obese women with NGT, consisting of 39 women with PCOS and 44 controls matched for BMI and HOMA-IR selected from the original study cohort. Plasma insulin and C-peptide $2 \mathrm{~h}$ following glucose loading remained higher in the PCOS group in the second cohort $(P<0.01)$. Androgen levels were also higher in women with PCOS $(P=0.001)$, whereas there were no differences in fasting insulin, glucose, or TG levels. Women with PCOS continued to demonstrate lower omentin-1 levels compared with those of the controls $(P<0.001$; Table 1 , Fig. 1B).

\section{Omentin-1 levels in groups stratified according to BMI and glucose tolerance status}

After dividing the subjects according to established BMI categories, the PCOS group demonstrated gradually decreasing plasma omentin-1 levels within the stratified BMI groups for all obesity levels compared with those in the control subjects (BMI $<18.5 \mathrm{~kg} / \mathrm{m}^{2}$, $P<0.001 ; 18.5-23 \mathrm{~kg} / \mathrm{m}^{2}, P=0.001$; and $\geq 23 \mathrm{~kg} / \mathrm{m}^{2}$, $P=0.002)$. However, after adjusting for age and HOMAIR, significant differences remained only in the lean and NW groups ( $P=0.001$; Fig. $2 \mathrm{~A})$. In both the control and the PCOS groups, increasing BMI level was associated with decreasing plasma omentin-1 level ( $P=0.017$ and $P=0.023$ respectively).

When stratified according to glucose tolerance status, the observed differences in omentin-1 level between the PCOS and the control groups remained significant in the NGR subgroup both before and after controlling for age and BMI $(P<0.001$; Fig. 2B). However, there were no differences in omentin-1 level between the PCOS and the control subjects in the IGR group (Fig. 2B). In control subjects, the IGR subgroup had significantly lower omentin-1 levels than did the NGR subgroup 
Table 1 Clinical, hormonal, and metabolic characteristics of women with PCOS and control subjects. Values expressed as mean \pm s.D., median (25th to 75 th percentile).

\begin{tabular}{|c|c|c|c|c|c|c|}
\hline & \multicolumn{3}{|c|}{ BMI-matched groups } & \multicolumn{3}{|c|}{ BMI- and HOMA-matched group } \\
\hline & $\operatorname{PCOS}(n=87)$ & Control $(n=53)$ & $P$ value & $\operatorname{PCOS}(n=39)$ & Control $(n=44)$ & $P$ value \\
\hline Age (year) & $26(22-29)$ & $26(24-31.5)$ & 0.019 & $25(22-28)$ & $26(24-29.7)$ & 0.019 \\
\hline $\mathrm{BMI}\left(\mathrm{kg} / \mathrm{m}^{2}\right)$ & $20.5(18.8-22.9)$ & $19.9(18.7-21.3)$ & NS & $19.59 \pm 1.64$ & $19.65 \pm 1.53$ & NS \\
\hline Fasting glucose $(\mathrm{mmol} / \mathrm{l})$ & $5.44(5.11-5.78)$ & $5.16(4.94-5.33)$ & $<0.001$ & $5.16(4.94-5.33)$ & $5.13(4.90-5.27)$ & 0.407 \\
\hline $2 \mathrm{~h}$ post glucose $(\mathrm{mmol} / \mathrm{l})$ & $6.30 \pm 1.72$ & $5.37 \pm 0.86$ & $<0.001$ & $5.37 \pm 0.79$ & $5.32 \pm 0.80$ & 0.875 \\
\hline Fasting C-peptide (nmol/l) & $0.85(0.59-1.11)$ & $0.73(0 . \overline{58}-0.79)$ & 0.002 & $0.68(0.54-0.89)$ & $0.72(0.60-0.79)$ & 0.925 \\
\hline $2 \mathrm{~h}$ post $\mathrm{C}$-peptide $(\mathrm{nmol} / \mathrm{l})$ & $2.64(2.14-4.37)$ & $1.68(1.20-2.43)$ & $<0.001$ & $2.76 \pm 1.25$ & $1.83 \pm 0.95$ & $<0.001$ \\
\hline Fasting insulin $(\mathrm{pmol} / \mathrm{l})$ & $75.0(54.0-99.0)$ & $69.2(51.4-82.9)$ & 0.057 & $55.9(47.4-76.4)$ & $66.7(50.5-83.8)$ & 0.371 \\
\hline $2 \mathrm{~h}$ post Insulin (pmol//l) & $260(177-499)$ & $157(100-202)$ & $<0.001$ & $200(142-326)$ & 147 (99.3-197) & 0.006 \\
\hline HOMA & $2.63(1.78-3.53)$ & $2.36(1.51-2.72)$ & 0.012 & $1.78(1.53-2.62)$ & $2.22(1.46-2.69)$ & NS \\
\hline Total cholesterol $(\mathrm{mmol} / \mathrm{l})$ & $4.77 \pm 0.79$ & $4.58 \pm 0.71$ & 0.178 & $4.66 \pm 0.66$ & $4.54 \pm 0.74$ & 0.437 \\
\hline LDL-cholesterol (mmol//) & $2.66 \pm 0.73$ & $2.41 \pm 0.57$ & 0.039 & $2.52 \pm 0.54$ & $2.36 \pm 0.55$ & 0.185 \\
\hline HDL-cholesterol (mmol/l) & $1.53(1.37-1.76)$ & $1.71(1.60-1.90)$ & 0.001 & $1.71 \pm 0.36$ & $1.78 \pm 0.29$ & 0.318 \\
\hline Triglyceride $(\mathrm{mmol} / \mathrm{l})$ & $0.86(0.69-1.21)$ & $0.71(0.55-0.89)$ & 0.001 & $0.78(0.61-0.97)$ & $0.69(0.55-0.88)$ & 0.080 \\
\hline LH (IU/I) & 8.69 (5.74-12.07) & $4.02(2.38-6.29)$ & $<0.001$ & $10.1(7.69-14.18)$ & $3.99(2.37-6.06)$ & $<0.001$ \\
\hline FSH (IÚ/I) & $4.62(3.39-5.74)$ & $4.41(3.26-6.15)$ & 0.782 & $4.82(3.91-5.94)$ & $4.26(3.18-6.17)$ & 0.430 \\
\hline $\mathrm{E}_{2}(\mathrm{pmol} / \mathrm{l})$ & $277(213-428)$ & $302(175-533)$ & 0.699 & $400.5 \pm 440.5$ & $356.9 \pm 224.0$ & 0.567 \\
\hline $17-\mathrm{OH}-\mathrm{P}(\mathrm{nmol} / \mathrm{l})$ & $4.55(3.33-6.36)$ & $3.33(2.36-7.58)$ & 0.05 & $4.24(3.03-6.36)$ & $3.33(2.45-7.73)$ & 0.403 \\
\hline Total testosterone $(\mathrm{nmol} / \mathrm{l})$ & $1.77 \pm 0.90$ & $0.97 \pm 0.45$ & $<0.001$ & $1.84 \pm 1.01$ & $1.01 \pm 0.45$ & $<0.001$ \\
\hline Free testosterone (pmol/l) & $5.20(2 . \overline{77}-7.99)$ & $2.15(1.63-3.47)$ & $<0.001$ & $3.99(2.19-7.18)$ & $2.15(1 . \overline{6} 0-3.47)$ & 0.001 \\
\hline DHEA-S $(\mu \mathrm{mol} / \mathrm{l})$ & $5.97 \pm 3.20$ & $4.39 \pm 1.99$ & 0.003 & $5.94 \pm 2.95$ & $4.27 \pm 1.88$ & 0.004 \\
\hline SHBG (nmol/l) & $65.84 \pm 35.29$ & $85.23 \pm 21.99$ & $<0.001$ & $67.37 \pm 26.76$ & $84.31 \pm 21.79$ & 0.004 \\
\hline Free androgen index & $8.95(5.17-17.87)$ & $3.67(2 . \overline{60}-5.59)$ & $<0.001$ & $8.74(5.69-13.91)$ & $4.19(2.39-5.60)$ & $<0.001$ \\
\hline Hs-CRP (mg/l) & $0.6(0.3-1.7)$ & $0.2(0.1-0.4)$ & $<0.001$ & $0.35(0.2-0.5)$ & $0.2(0.1-0.4)$ & 0.012 \\
\hline Adiponectin $(\mu \mathrm{g} / \mathrm{ml})$ & $6.91(4.84-9.93)$ & $8.51(5.38-10.94)$ & 0.06 & $9.11 \pm 3.69$ & $9.40 \pm 4.52$ & 0.752 \\
\hline Omentin-1 $(\mu \mathrm{g} / \mathrm{ml})$ & $0.41(0.34-0.54)$ & $0.55(0.44-0.73)$ & $<0.001$ & $0.454 \pm 0.102$ & $0.632 \pm 0.192$ & $<0.001$ \\
\hline
\end{tabular}

PCOS, polycystic ovary syndrome; BMI, body mass index; HOMA, homeostasis model assessment; $\mathrm{E}_{2}$, estradiol; 17-OH-P, 17-OH-progesterone; DHEA-S, DHEA-sulfate; SHBG, sex hormone-binding globulin; FAI, free androgen index; LDL, low-density lipoprotein; HDL, high-density lipoprotein; hs-CRP, high sensitivity C-reactive protein; NS, not significant.

$(P=0.025)$. There were significant differences in omentin-1 level in the PCOS group according to glucose tolerance status.

\section{Associations of omentin-1 level with clinical/biochemical variables}

Bivariate correlation analysis indicated that serum omentin-1 concentration was negatively correlated with BMI, many of the indices of glucose metabolism, IR, and androgen levels and was positively correlated with adiponectin and SHBG levels (Table 2).

In the second cohort of non-obese women with NGT, significant correlations were found between plasma omentin-1 level and BMI $(r=-0.288, P=0.008), 2 \mathrm{~h}$ postprandial C-peptide level $(r=-0.268, P=0.022)$, and androgen levels (free testosterone; $r=-0.332$, $P=0.002$, FAI; $r=-0.279, P=0.018$; Table 2).

\section{Relationships between variables associated with PCOS and omentin-1 level}

To determine contributing factors other than BMI and IR on plasma omentin-1 levels in women with PCOS, separate analyses were performed in a second cohort of non-obese women with NGT. BMI and HOMA-IR were not significantly different between subjects with PCOS and controls in this second cohort; therefore, other factors may contribute to circulating omentin-1 level.

Because androgen levels were higher in women with PCOS compared with those in control subjects and continued to demonstrate significant negative correlations with omentin-1 level in the second cohort subjects (Fig. 3A), androgen levels were considered to be potential contributors to plasma omentin-1 level. To further investigate this possibility, we stratified subjects of the second cohort into four groups according to quartile of free testosterone. Age, BMI, and HOMA-IR were not significantly different among the four subgroups (data not shown). Mean values of omentin-1 significantly decreased from the lowest to the highest quartile group $(P=0.028 ;$ Fig. $3 \mathrm{~B})$. When post hoc analyses were performed among groups, the relationship between plasma omentin-1 level and free testosterone was significantly higher in the lowest quartile group than in the highest quartile group $(P=0.017$; Fig. 3B).

When logistical regression analysis was performed to determine the predictors of PCOS with omentin-1 level, age, HOMA, BMI, and free testosterone included as potential factors, the odds ratios for PCOS were -7.099 $(P<0.001)$ with omentin-1, $-0.094(P=0.054)$ with age, $0.699(P=0.012)$ with HOMA-IR, 1.458 $(P=0.001)$ with free testosterone, and -0.226 $(P=0.051)$ for BMI (data not shown). 

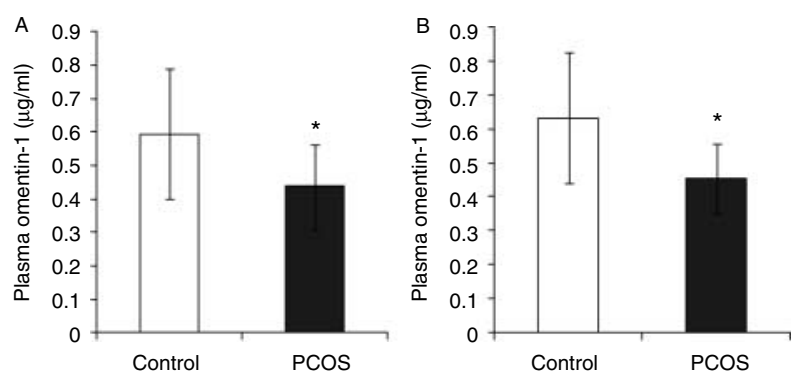

Figure 1 Plasma omentin-1 levels in women with PCOS compared with BMI-matched controls $(\mathrm{A})$ and in women with PCOS compared with BMI- and HOMA-matched controls in non-obese and NGT group (B). Data are adjusted mean \pm S.E.M. Plasma omentin-1 levels were log transformed before analysis. The black bar represents the PCOS cohort and the white bar represents controls. Significance was determined by ANCOVA, controlling for age. ${ }^{*} P<0.001$.

\section{Discussion}

Women with PCOS have multiple risk factors for diabetes and CVD, both of which are considered major long-term health risks (2). Because PCOS is a heterogeneous disease, diverse mechanisms may be involved in its pathogenesis. In an attempt to further investigate the role of adipokines in PCOS, many researchers have examined the relationships between adipokines and PCOS characteristics, such as adiposity, IR, and androgen levels. These studies have produced conflicting results. Therefore, this study of circulating omentin1 level was carried out to better understand the regulation and role of omentin-1 in the setting of PCOS.

In agreement with a previous report $(18,19)$, this study demonstrated that omentin-1 levels are decreased in women with PCOS compared with those in BMImatched control subjects and that these levels are negatively correlated with BMI, glucose, insulin, and androgen levels. In this study, $2 \mathrm{~h}$ postprandial glucose, C-peptide, and insulin levels were more strongly associated with omentin-1 level than were fasting values. Additionally, we report for the first time that significantly lower plasma omentin-1 levels were detected in non-obese women with PCOS and NGT compared with those in BMI- and HOMA-IR-matched control subjects. Moreover, $2 \mathrm{~h}$ postprandial C-peptide and androgen levels continued to demonstrate negative correlations with omentin-1 level in the non-obese cohort with NGT, as in the overall study cohort. Tan et al. (18) previously suggested that hyperinsulinemic induction in healthy subjects significantly reduced plasma omentin-1 levels; we suggest that postprandial hyperinsulinemia and hyperglycemia may contribute more to decreased omentin-1 levels in PCOS subjects than do these factors in the fasting state.

Batista et al. (14) reported that plasma omentin-1 levels are decreased in healthy, obese subjects. This finding is in compliance with our study results.
Furthermore, reduced omentin-1 levels in the PCOS group were consistently significant, even after controlling for age and HOMA-IR. When omentin-1 levels were compared among groups divided by obesity status, significant differences in omentin-1 between PCOS and control groups disappeared in the overweight/obese group after controlling for age and HOMA. We hypothesize that omentin-1 level in overweight/obese subjects is more influenced by other factors than it is by PCOS and that omentin-1 level is regulated by more complex mechanisms in normoweight/lean subjects.

Omentin is a recently identified polypeptide hormone that is known to increase insulin sensitivity in human adipocytes (13). In this study, we stratified the subjects into NGR and IGR groups according to glucose metabolism status. As in a previous study (24), subjects in the IGR subgroup had decreased serum omentin-1
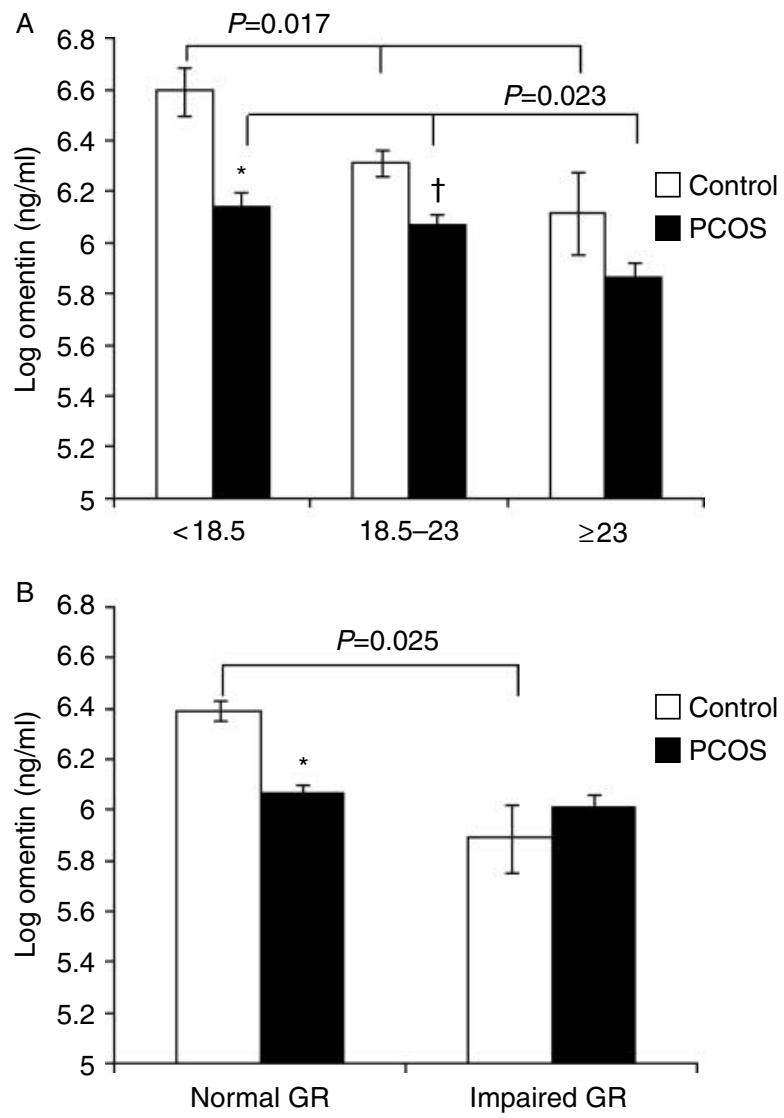

Figure 2 Plasma omentin-1 levels in women with PCOS compared with controls in BMI-stratified analysis $(A)$ and in glucose metabolism status stratified analysis (B). Values represent adjusted mean \pm S.E.M. Plasma omentin-1 levels were log transformed before analysis. $P$ values between control and PCOS in each obesity status were determined by ANCOVA, controlling age and HOMA $\left({ }^{\star} P=0.001\right.$ and ${ }^{\dagger} P<0.001$ respectively). Three groups for BMI stratification in both control and PCOS show linear trend $(P=0.019$ and $P=0.03$ respectively (A)). $P$ values between control and PCOS in each glucose metabolism status are adjusted for age, BMI. ${ }^{*} P<0.001(\mathrm{~B})$. 
Table 2 Plasma omentin-1 levels correlations with baseline parameters in all subjects and NW and NGT subgroup.

\begin{tabular}{lrrrrr}
\hline & \multicolumn{2}{c}{ All subjects } & & \multirow{2}{*}{ NW and NGT group } \\
\cline { 2 - 3 } \cline { 5 - 6 } & CC $(\gamma)$ & $P$ value & & CC $(\gamma)$ & $P$ value \\
\hline BMI & -0.407 & $<0.001$ & & -0.288 & 0.008 \\
Fasting glucose & -0.183 & 0.031 & & 0.038 & 0.736 \\
2 h post glucose & -0.197 & 0.02 & & 0.059 & 0.594 \\
Fasting C-peptide & -0.312 & $<0.001$ & & -0.111 & 0.347 \\
2 h post C-peptide & -0.335 & $<0.001$ & & -0.268 & 0.022 \\
Fasting insulin & -0.130 & 0.125 & & 0.144 & 0.195 \\
2 h post insulin & -0.229 & 0.007 & & -0.058 & 0.602 \\
HOMA & -0.151 & 0.076 & & 0.158 & 0.158 \\
HDL-cholesterol & 0.273 & 0.001 & & 0.102 & 0.360 \\
Triglycerides & -0.250 & 0.003 & & -0.009 & 0.938 \\
SHBG & 0.209 & 0.019 & & 0.194 & 0.103 \\
Free testosterone & -0.322 & $<0.001$ & & -0.332 & 0.002 \\
Total testosterone & -0.228 & 0.007 & & -0.268 & 0.014 \\
FAl & -0.261 & 0.003 & & -0.279 & 0.018 \\
Adiponectin & 0.312 & $<0.001$ & & 0.126 & 0.257 \\
\hline
\end{tabular}

NW; non-obese, NGT; normal glucose tolerance; CC, correlation coefficient; BMI, body mass index; HOMA, homeostasis model assessment; HDL, high-density lipoprotein; SHBG, sex hormone-binding globulin; FAl, free androgen index.

levels compared with those in the NGR subgroup in healthy control subjects. Our data are in agreement with those of Tan et al. (25), who found that omentin is important for glucose metabolism. When omentin-1 levels were compared between control and PCOS subjects in the NGR and IGR groups, significant differences were observed in the NGR group between PCOS and control subjects, both before and after controlling for age and BMI. However, differences in omentin-1 level according to PCOS status were not significant in the IGR group. We speculate that factors other than BMI, IR, and the degree of glucose intolerance may influence circulating omentin-1 level in women with PCOS, especially in non-obese women with NGT.

In this study, we found that increased free testosterone levels accompanied decreased plasma omentin-1 level. Given the higher concentration of circulating androgens common in PCOS subjects and the negative correlation between omentin-1 and androgen levels in the second study cohort, we propose androgen concentration as one of the most influential factors contributing to reduced omentin-1 level in women with PCOS. Batista et al. (14) reported increased omentin-1 concentrations in lean women compared with those in lean men in a genetically homogeneous population, supporting the knowledge that many adipokines exhibit sexual dimorphism. Additionally, androgens are reported to promote central adiposity and visceral adipose deposition and to exert direct effects on adipocytes in females $(26,27)$. It is interesting to note that the associations of omentin-1 level with metabolic variables were similar to those described for adiponectin, suggesting common insulin-sensitizing activities $(14,18)$.
In agreement with previous reports, circulating total plasma adiponectin levels were positively correlated with plasma omentin-1 values in this study. The inverse relationships between obesity and both omentin and adiponectin suggest similar regulations for these proteins. In PCOS subjects, O'Connor et al. (28) suggested that the combined effects of waist-hip ratio (WHR) and free testosterone level demonstrated influences on adiponectin levels independent of BMI and IR. This group also found that increased expression of androgen receptor mRNA in subcutaneous adipose tissue obtained from women with PCOS supported the possibility that increased androgens could influence the adipocyte function in these groups. Although androgens may not be the principal factor in regulation of omentin-1, they may contribute to decreased omentin-1 levels in women with PCOS. We speculate that decreased omentin-1 level may influence obesity and IR in women with PCOS. Because this was a crosssectional study, the cause-and-effect relationship between omentin-1 and androgen levels cannot be determined. Prospective studies are needed to clarify this relationship.

A limitation of this study is that body fat distribution or WHR, which is known to reflect the visceral adiposity, was not evaluated. It has been suggested that differences in adipose tissue distribution may influence the secretion of adipokines $(11,29)$, BMI may be a relatively insensitive index of the distribution of adipose mass, and omentin is an adipokine preferentially expressed in visceral adipose tissue. This study did not address adipose tissue deposition patterns in the study subjects; it is possible that these differences may influence the study conclusions. Another limitation is that, since the data for the normal omentin levels in thin, normal weight women in their reproductive age in the literature were not sufficient or appropriate to use as the reference $(30,31)$, we could not determine whether the omentin levels in control subjects in our study are appropriate or not, neither are the omentin levels in PCOS patients. However, as these

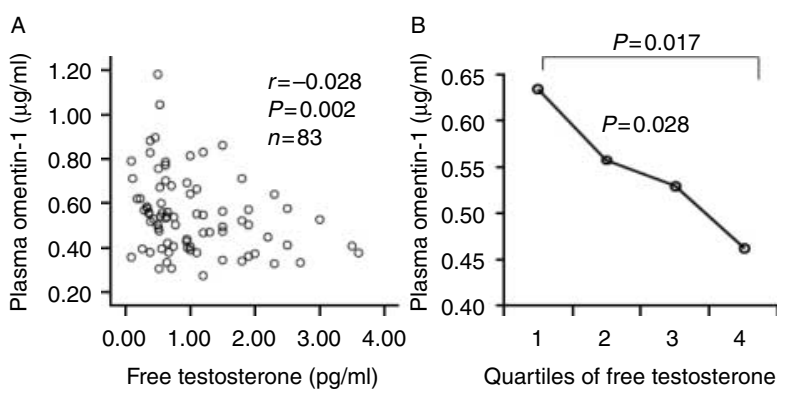

Figure 3 Relationship between plasma omentin-1 levels and free testosterone in NW and NGT women. Association between free testosterone and plasma omentin-1 (A). Omentin-1 across quartiles of free testosterone (B). Data are mean values. $P$ value for quartiles of free testosterone between the four groups was determined by one-way ANOVA using the Tukey test $(P=0.028)$. 
analyses were compared between those with and without PCOS, we could assume our results as an additive data for the omentin levels in women in their reproductive age.

In conclusion, postprandial hyperinsulinemia and hyperglycemia contribute more to reduced omentin-1 level in PCOS subjects than do the fasting values. Although omentin-1 is an adipokine closely associated with obesity, IR, and glucose metabolism, androgen levels also demonstrated significant interactions with decreased omentin-1 level in PCOS subjects. Based on these results, we suggest that the regulation of omentin1 production in adipose tissue is multi-factorial, and reduced omentin-1 and increased androgen levels in PCOS may contribute to the pathogenesis of IR and obesity in the setting of this disease. Further investigation is needed to further elucidate mechanisms underlying regulation of omentin-1 and to determine whether a decreased omentin-1 level can serve as a marker for the development of diabetes and obesity in PCOS.

\section{Declaration of interest}

The authors declare that there is no conflict of interest that could be perceived as prejudicing the impartiality of the research reported.

\section{Funding}

This work was supported by research grants from the IN-SUNG Foundation for Medical Research.

\section{References}

1 Azziz R, Woods KS, Reyna R, Key TJ, Knochenhauer ES \& Yildiz BO. The prevalence and features of the polycystic ovary syndrome in an unselected population. Journal of Clinical Endocrinology and Metabolism 200489 2745-2749. (doi:10.1210/jc.2003032046)

2 Rotterdam ESHRE/ASRM-Sponsored PCOS Consensus Workshop Group. Revised 2003 consensus on diagnostic criteria and longterm health risks related to polycystic ovary syndrome. Fertility and Sterility $2004 \mathbf{8 1} 19-25$.

3 Wild S, Pierpoint T, McKeigue P \& Jacobs H. Cardiovascular disease in women with polycystic ovary syndrome at long-term follow-up: a retrospective cohort study. Clinical Endocrinology $2000 \mathbf{5 2}$ 595-600. (doi:10.1046/j.1365-2265.2000.01000.x)

4 Christian RC, Dumesic DA, Behrenbeck T, Oberg AL, Sheedy PF II \& Fitzpatrick LA. Prevalence and predictors of coronary artery calcification in women with polycystic ovary syndrome. Journal of Clinical Endocrinology and Metabolism 200388 2562-2568. (doi:10.1210/jc.2003-030334)

5 Carmina E, Napoli N, Longo RA, Rini GB \& Lobo RA. Metabolic syndrome in polycystic ovary syndrome (PCOS): lower prevalence in southern Italy than in the USA and the influence of criteria for the diagnosis of PCOS. European Journal of Endocrinology 2006154 141-145. (doi:10.1530/eje.1.02058)

6 Carmina E \& Lobo RA. Polycystic ovary syndrome (PCOS): arguably the most common endocrinopathy is associated with significant morbidity in women. Journal of Clinical Endocrinology and Metabolism $1999 \mathbf{8 4}$ 1897-1899. (doi:10.1210/jc.84.6. 1897)
7 Ovalle F \& Azziz R. Insulin resistance, polycystic ovary syndrome, and type 2 diabetes mellitus. Fertility and Sterility $2002 \mathbf{7 7}$ 1095-1105. (doi:10.1016/S0015-0282(02)03111-4)

8 Acien P, Quereda F, Matallin P, Villarroya E, Lopez-Fernandez JA, Acien M, Mauri M \& Alfayate R. Insulin, androgens, and obesity in women with and without polycystic ovary syndrome: a heterogeneous group of disorders. Fertility and Sterility 199972 32-40. (doi:10.1016/S0015-0282(99)00184-3)

9 Yamauchi T, Kamon J, Waki H, Terauchi Y, Kubota N, Hara K, Mori Y, Ide T, Murakami K, Tsuboyama-Kasaoka N, Ezaki O, Akanuma Y, Gavrilova O, Vinson C, Reitman ML, Kagechika H, Shudo K, Yoda M, Nakano Y, Tobe K, Nagai R, Kimura S, Tomita M, Froguel P \& Kadowaki T. The fat-derived hormone adiponectin reverses insulin resistance associated with both lipoatrophy and obesity. Nature Medicine 2001 7 941-946. (doi:10.1038/90984)

10 Carmina E, Orio F, Palomba S, Cascella T, Longo RA, Colao AM, Lombardi G \& Lobo RA. Evidence for altered adipocyte function in polycystic ovary syndrome. European Journal of Endocrinology 2005 152 389-394. (doi:10.1530/eje.1.01868)

11 Wajchenberg BL. Subcutaneous and visceral adipose tissue: their relation to the metabolic syndrome. Endocrine Reviews 200021 697-738. (doi:10.1210/er.21.6.697)

12 Thorne A, Lonnqvist F, Apelman J, Hellers G \& Arner P. A pilot study of long-term effects of a novel obesity treatment: omentectomy in connection with adjustable gastric banding. International Journal of Obesity and Related Metabolic Disorders 2002 26 193-199. (doi:10.1038/sj.ijo.0801871)

13 Yang RZ, Lee MJ. Hu H, Pray J. Wu HB, Hansen BC, Shuldiner AR, Fried SK, McLenithan JC \& Gong DW. Identification of omentin as a novel depotspecific adipokine in human adipose tissue: possible role in modulating insulin action. American Journal of Physiology. Endocrinology and Metabolism 2006290 1253-1261. (doi:10. 1152/ajpendo.00572.2004)

14 Souza Batista CM, Yang RZ, Lee MJ, Glynn NM, Yu DZ, Pray J, Ndubuizu K, Patil S, Schwartz A, Kligman M, Fried SK, Gong DW, Shuldiner AR, Pollin TI \& McLenithan JC. Omentin plasma levels and gene expression are decreased in obesity. Diabetes $2007 \mathbf{5 6}$ 1655-1661. (doi:10.2337/db06-1506)

15 Chae SJ, Kim JJ, Choi YM, Hwang KR, Jee BC, Ku SY, Suh CS, Kim SH, Kim JG \& Moon SY. Clinical and biochemical characteristics of polycystic ovary syndrome in Korean women. Human Reproduction 200823 1924-1931. (doi:10.1093/hum$\mathrm{rep} / \mathrm{den} 239$ )

16 Barber TM, Wass JA, McCarthy MI \& Franks S. Metabolic characteristics of women with polycystic ovaries and oligoamenorrhoea but normal androgen levels: implications for the management of polycystic ovary syndrome. Clinical Endocrinology 200766 513-517.

17 Belosi C, Selvaggi L, Apa R, Guido M, Romualdi D, Fulghesu AM \& Lanzone A. Is the PCOS diagnosis solved by ESHRE/ASRM 2003 consensus or could it include ultrasound examination of the ovarian stroma? Human Reproduction 200621 3108-3115. (doi:10.1093/humrep/del306)

18 Tan BK, Adya R, Farhatullah S, Lewandowski KC, O'Hare P, Lehnert H \& Randeva HS. Omentin-1, a novel adipokine, is decreased in overweight insulin-resistant women with polycystic ovary syndrome: $e x$ vivo and in vivo regulation of omentin-1 by insulin and glucose. Diabetes 200857 801-808. (doi:10.2337/ db07-0990)

19 Tan BK, Adya R, Farhatullah S, Chen J, Lehnert H \& Randeva HS. Metformin treatment may increase omentin-1 levels in women with polycystic ovary syndrome. Diabetes 201059 3023-3031. (doi:10.2337/db10-0124)

20 Hatch R, Rosenfield RL, Kim MH \& Tredway D. Hirsutism: implications, etiology, and management. American Journal of Obstetrics and Gynecology $1981 \mathbf{1 4 0} 815-830$.

21 The International Association for the Study of Obesity and the International Obesity Task Force. The Asia-Pacific perspective: redefining obesity and its treatment. Australia: IASO and IOTF, 2000. 
22 Sacks DB, Arnold M, Bakris GL, Bruns DE, Horvath AR, Kirkman MS, Lernmark A, Metzger BE \& Nathan DM. Guidelines and recommendations for laboratory analysis in the diagnosis and management of diabetes mellitus. Diabetes Care 201134 e61-e99. (doi:10.2337/dc11-9998)

23 Matthews DR, Hosker JP, Rudenski AS, Naylor BA, Treacher DF \& Turner RC. Homeostasis model assessment: insulin resistance and beta-cell function from fasting plasma glucose and insulin concentrations in man. Diabetologia 198528 412-419. (doi:10. 1007/BF00280883)

24 Pan HY, Guo L \& Li Q. Changes of serum omentin-1 levels in normal subjectsand in patients with impaired glucose regulation and with newly diagnosed and untreated type 2 diabetes. Diabetes Research and Clinical Practice 201088 29-33. (doi:10.1016/ j.diabres.2010.01.013)

25 Tan BK, Pua S, Syed F, Lewandowski KC, O'Hare JP \& Randeva HS. Decreased plasma omentin-1 levels in type 1 diabetes mellitus. Diabetic Medicine 200825 1254-1255. (doi:10.1111/j.14645491.2008.02568.x)

26 Xu A, Chan KW, Hoo RL, Wang Y, Tan KC, Zhang J, Chen B, Lam MC, Tse C, Cooper GJ \& Lam KS. Testosterone selectively reduces the high molecular weight form of adiponectin by inhibiting its secretion from adipocytes. Journal of Biological Chemistry $2005 \mathbf{2 8 0}$ 18073-18080. (doi:10.1074/jbc.M414 231200)

27 Blouin K, Boivin A \& Tchernof A. Androgens and body fat distribution. Journal of Steroid Biochemistry and Molecular Biology 2008108 272-280. (doi:10.1016/j.jsbmb.2007.09.001)
28 O'Connor A, Phelan N, Tun TK, Boran G, Gibney J \& Roche HM. High-molecular-weight adiponectin is selectively reduced in women with polycystic ovary syndrome independent of body mass index and severity of insulin resistance. Journal of Clinical Endocrinology and Metabolism 2010 95 1378-1385. (doi:10. 1210/jc.2009-1557)

29 Masaki T, Chiba S, Yasuda T, Tsubone T, Kakuma T, Shimomura I, Funahashi T, Matsuzawa Y \& Yoshimatsu H. Peripheral, but not central, administration of adiponectin reduces visceral adiposity and upregulates the expression of uncoupling protein in agouti yellow (Ay/a) obese mice. Diabetes 200352 2266-2273. (doi:10. 2337/diabetes.52.9.2266)

30 Auguet T, Quintero Y, Riesco D, Morancho B, Terra X, Crescenti A, Broch M, Aguilar C, Olona M, Porras JA, Hernandez M, Sabench F \& Richart C. New adipokines vaspin and omentin. Circulating levels and gene expression in adipose tissue from morbidly obese women. BMC Medical Genetics 201112 60. (doi:10.1186/14712350-12-60)

31 Moreno-Navarrete JM, Catalán V, Ortega F, Gómez-Ambrosi J, Ricart W, Frühbeck G \& Fernández-Real JM. Circulating omentin concentration increases after weight loss. Nutrition $\mathcal{E}$ Metabolism 20107 27. (doi:10.1186/1743-7075-7-27)

Received 28 April 2011

Revised version received 20 August 2011

Accepted 24 August 2011 